\section{Difficult IUD insertions}

I read with interest Dr Isabel Draper's letter ${ }^{1}$ regarding difficult intrauterine device (IUD) insertions published in the January 2008 issue of this Journal. I agree with Dr Draper's views and would like to share my experience on this subject.

I am an instructing doctor and do two IUD/intrauterine system (IUS) training clinics every week. One training clinic is at The Palatine Centre in Manchester and the other is a Gynaecology Tier 2 clinic in Stockport with facilities for on-site ultrasound scanning for difficult IUD/IUS insertions/removals. On an average five patients are seen in each clinic for IUD/IUS insertions.

Nearly $30 \%$ of patients I see are under the age of 25 years and nulliparous. In my experience I have found that insertion of TT380 Slimline ${ }^{\circledR}$, TCu380 ${ }^{\circledR}$, QuickLoad ${ }^{\circledR}$ or T- Safe $380 A^{\circledR}$, which are current recommended gold standards, can be at times difficult and painful to insert in this group of patients. I agree with the author's comments that insertion of the IUS can also be challenging in this group of women.

I find the following methods helpful in reducing the discomfort associated with IUD/IUS insertions.

1 Injection of local anaesthetic directly into the cervix (intracervical block) at the 3,6,9 and 12 o'clock position is very effective. A 27 gauge dental syringe is used to inject 3\% Mepivacaine (Scandonest ${ }^{\circledR}$ ) or Articaine ${ }^{\circledR}$ with adrenaline $\left(\right.$ Septanest $\left.{ }^{\circledR}\right)$, which is available in cartridges. In order to divert the women's attention I usually ask the patient to cough at the time of injection.

2 Anaesthetic gel such as Instillagel ${ }^{\circledR}$ (lidocaine $2 \%$ and chlorhexidine gluconate solution $0.25 \%)$ used with Instillaquill ${ }^{\circledR}$ applied on the ectocervix and directly into the endocervical canal takes up to 5 minutes to work. Therefore I rely on its lubricant properties in enhancing ease of uterine instrumentation.

3 Topical application of lidocaine ointment $(5 \%)$ on the ectocervix

There is a lack of randomised controlled trials investigating the use of topical or intracervical anaesthesia during IUD/IUS insertions.

Methods used to aid clinicians in dilating the cervical os if resistance is encountered are listed below.

1 A plastic disposable graduated uterine sound and dilator is available from Durbin Sales. Dilatation up to $5.5 \mathrm{~mm}$ can be achieved with this disposable plastic instrument that has a graduated cervical dilator at one end and a sound at the other end. It is marked at $1 \mathrm{~cm}$ intervals, and the dilator end is tapered with gradual increase in width to $5.5 \mathrm{~mm}$ and has a gentle curve. In my experience it is easy to use compared to the metal Hegars dilators.

2 Vaginal use of misoprostol $(200 \mu \mathrm{g})$ inserted 3 hours prior to IUD/IUS insertion softens the cervix. This may cause some vaginal bleeding and cramps, and due to the risk of teratogenicity should be used during a period or a reliable method of contraception should be advised during that cycle.

Previous Caesarean section is a risk factor for perforation. ${ }^{2}$ Risk of perforation is directly proportional to the degree of difficulty encountered during insertion, clinician experience and technique.

I can foresee that these potential difficult cases will be referred to specialist clinics or will be done by experienced practitioners.

I share Dr Draper's concerns about trainees who would have to learn in this environment and the need for more training sessions to enable them to achieve adequate skills.

Manju Navani, MFSRH, MRCOG Associate Specialist, Stockport Primary Care Trust, Contraception and Sexual Health,

Adswood Clinic, Stockport, UK.

E-mail:manju.navani@yahoo.co.uk
References

Draper IB. Difficult IUD insertions [Letter]. J Fam Plann Reprod Health Care 2008; 34: 67

Chi I, Feldbum PJ, Rogers SM. IUD-related uterine perforation: an epidemiological analysis of a rare event using an international data set. Adv Contracept Del Systems 1984; 5: 123-130.

\section{Reply}

I agree with Dr Navani1 that the plastic disposable sound and dilator seems more satisfactory than traditional metal dilators. A technique I use with Instillage ${ }^{\circledR}$ that seems to make this method of local anaesthetic more effective is to keep the speculum in place for the 5 minutes after applying it (with due apologies to the woman for the indignity!) so the cervix is bathed in the gel that pools in the jaws of the speculum. I was interested to read of the use of misoprostol in this situation, which I was not aware of.

Some innovative ideas have obviously evolved in response to this problem. Perhaps future article for the journal could be a summary of these, seeing as I am obviously not alone in experiencing the problem.

Isabel B Draper, MRCGP, FFSRH

General Practitioner, Whitehall Medical

Practice, Rugby, UK.

E-mail:IBD@doctors.org.uk

\section{Reference}

Navani M. Difficult IUD insertions [Letter]. J Fam Plann Reprod Health Care 2008; 34: 135 .

\section{Removal of copper-bearing IUDs in asymptomatic patients}

We read with interest the case report regarding the migration of an intrauterine device (IUD) in an asymptomatic woman post-insertion. We have also recently had a case of IUD migration in an asymptomatic patient. She had a copper-bearing IUD inserted 10 weeks after normal vaginal delivery that was uncomplicated. The patient had been fully counselled regarding contraceptive choices, and was still breastfeeding at this time. As per the World Health Organization Medical Eligibility Criteria for Contraceptive Use (WHOMEC), the IUD was inserted more than 4 weeks postpartum. ${ }^{2}$

A review appointment 2 months later found that the strings of the IUD were absent. A pelvic ultrasound demonstrated that the IUD was no longer in the uterine cavity and an abdominal X ray confirmed the presence of the IUD in the abdominal cavity.

Following usual clinical practice guidelines, laparoscopy was performed to extract the device. This occurred 4 months after initial insertion. Figures 1 and 2 show that the IUD was already encased in adhesions. Fortunately, this IUD was clearly visualised at the time of the procedure and was easily retrieved via the laparoscopic approach (Figure 3). The patient herself had no clinical symptoms, and chose to have another copper-containing IUD inserted during the same procedure. She was reviewed again 6 weeks postoperatively having had her first menses postpartum without issues.

Markovitch et al. have argued that extrauterine IUDs need not be surgically removed in well patients. Their series of three patients had no adhesions. Two were asymptomatic, and one had lower abdominal pain. They speculate that adhesions may be perhaps caused by the initial inflammatory or infective process and may not progress. They also contend that the effects of the copper in IUDs are not definitively proven, and that surgery may also inflict greater harm. ${ }^{4}$

However, the patient described by Jatti et al. had a significant complication with a peritoneal abscess, and yet was relatively well. ${ }^{1}$ In our patient, the IUD was already enveloped in adhesions despite the short time duration within the pelvic cavity.
Markovitch et al. suggested a conservative approach in asymptomatic patients pending results from research in animal models. ${ }^{4}$ However, previous animal studies have demonstrated that adhesions from copper-bearing devices can cause severe adhesions involving necrosis and have greater fibrotic reactions than non-medicated IUDs. 5,6

We believe that the potential of complicated sequelae arising from the presence of a copperbearing IUD outweigh the benefits of a conservative approach. Minimally invasive methods to remove translocated IUDs are becoming the mainstay approach, with alternatives for locating the IUD that is not easily visualised in order to reduce conversion to laparotomy, thus decreasing patient morbidity. ${ }^{7}$ Extrauterine IUDs should therefore be removed even in the asymptomatic patient.

\section{Laura Lee, MBBS}

Registrar, Department of Obstetrics and Gynaecology, Royal Women's Hospital, Melbourne, Australia

Alex Ades, MD, FRANZCOG

Consultant Obstetrician and Gynaecologist, Department of Obstetrics and Gynaecology, Royal Women's Hospital, Melbourne, Australia

W Catarina Ang, MBBS, FRANZCOG Consultant, Gynaecologist and Laparoscopic Surgeon, Department of Obstetrics and Gynaecology, Royal Women's Hospital, Melbourne, Australia.

E-mail: catarina.ang@rwh.org.au
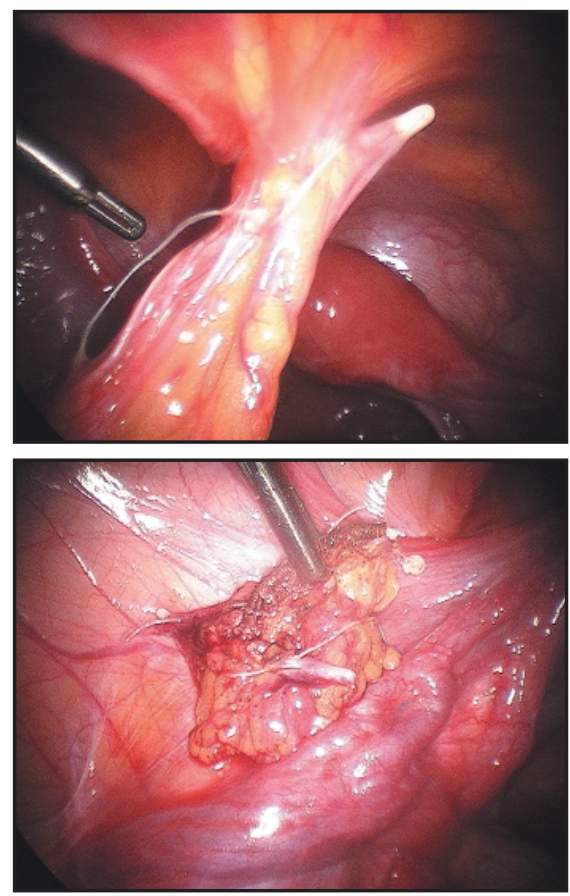

Figures 1 and 2 The intrauterine device is cased in adhesions 4 months following initial insertion

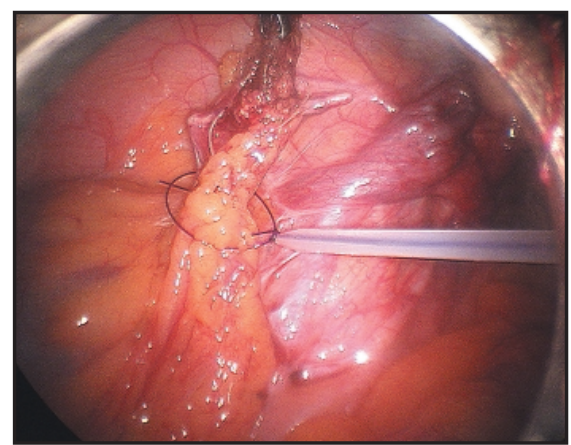

Figure 3 Removal of the intrauterine device using a laparoscopic approach 